\title{
Estimated Flood Flows in the Lake Tahoe Basin, California and Nevada
}

\section{Introduction}

Lake Tahoe, the largest alpine lake in North America, covers about 192 square miles $\left(\mathrm{mi}^{2}\right)$ of the $506-\mathrm{mi}^{2}$ Lake Tahoe Basin, which straddles the border between California and Nevada (Fig. 1). In cooperation with the Nevada Department of Transportation (NDOT), the U.S. Geological Survey (USGS) estimates the flood frequencies of the streams that enter the lake. Information about potential flooding of these streams is used by NDOT in the design and construction of roads and highways in the Nevada portion of the basin. The stream-monitoring network in the Lake Tahoe Basin is part of the Lake Tahoe Interagency Monitoring Program (LTIMP), which combines the monitoring and research efforts of various Federal, State, and regional agencies, including both USGS and NDOT.

The altitude in the basin varies from 6,223 feet (ft) at the lake's natural rim to over $10,000 \mathrm{ft}$ along the basin's crest. Precipitation ranges from 40 inches per year (in/yr) on the eastern side to $90 \mathrm{in} / \mathrm{yr}$ on the western side (Crippen and Pavelka, 1970). Most of the precipitation comes during the winter months as snow. Precipitation that falls from June through September accounts for less than 20 percent of the annual total.

\section{Methods}

The estimated magnitude of peak flows at 50- and 100-year recurrence intervals was determined for 46 sites in 21 watersheds that cover about $177 \mathrm{mi}^{2}$ of the Lake Tahoe Basin (Fig. 1; Table 1). The drainage areas of the 46 sites range in size from $0.63 \mathrm{mi}^{2}$ at Eagle Rock Creek near Stateline, Nev., (site 40) to $54.0 \mathrm{mi}^{2}$ at Upper Truckee River at South Lake Tahoe, Calif. (site 4). Expected peak flows were determined using five methods (refer to Table 1 footnotes for the method used to estimate discharge at each site).

Method 1 uses a USGS computer model (W.O. Thomas and others, U.S. Geological Survey, written commun., 1998) that is based on statistical flood-frequency analysis of annual peak-flow records according to the U.S. Interagency Advisory Committee on Water Data (1982) guidelines. This method employs the Pearson Type III distribution with $\log$ transformation of the data as the base method for flood-flow frequency. Method 2 is a variation of method 1 in which historic peaks are considered (W.O. Thomas and others, U.S. Geological Survey, written commun., 1998). This option modifies the length of the historic return period. Method 3 is a two-station comparison as presented in U.S. Interagency Advisory Committee on Water Data (1982) guidelines. This method is used to adjust the logarithmic mean and standard deviation of a short record on the basis of a regression analysis with a nearby long-term record.

Because methods 1-3 use annual peaks to determine the estimated peak discharge, better estimates are obtained by incorporating more years of data. The range in the period of record for the 20 sites at which these methods were used ranged from 10 years at seven sites to 40 years at two sites, with an average period of record of 18 years. The years for which data were collected at each site are listed in Table 1.

Method 4 estimates the magnitude of peak-flood discharge as a generalized least-square regression equation determined for the eastern Sierra (Thomas and others, 1997, p. 45). This equation uses drainage area, mean basin elevation, and latitude of the site in decimal degrees. In this method the period of record is not important.

Method 5 is an equation presented in Thomas and others (1997, p. 14) that was used to determine flood-frequency relations at sites near gaged sites on the same stream. This equation is based on the relation of the discharge at the gaged site and the drainage area of the two sites. Ideally, the drainage-area ratio should be approximately between 0.5 and 1.5. In methods 4 and 5 the period of record for the 24 sites ranged

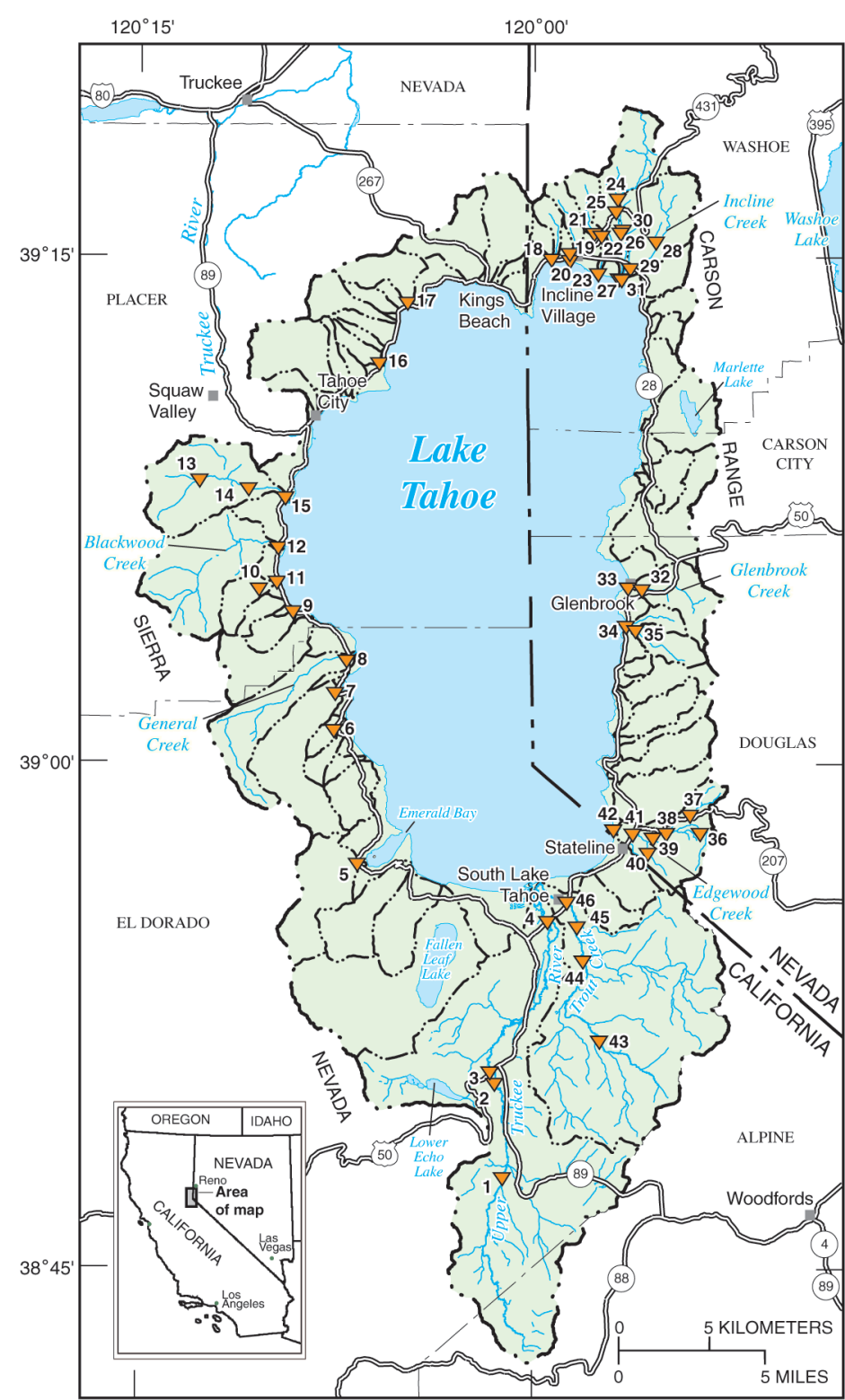

Base from U.S. Geological Survey digital data, 1:24,000 and 1:100,000,1969-85. Universal Transverse Mercator projection, Zone 11

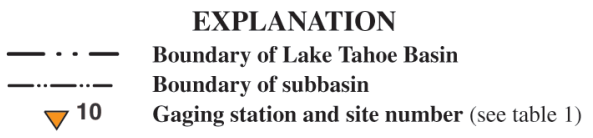

Figure 1. Geographic setting, hydrologic basins, and selected gaging stations in the Lake Tahoe Basin, California and Nevada

from 2 years at four sites to 15 years at one site, with an average period of record of 7 years. At sites 41 and 42 peak flows were not determined because these sites are affected by regulation.

The peak flows published in this report vary from -24 to 9 percent of the values published in Rowe and others (1998). These differences in estimated peak values can be explained either by the additional three years of data or by the methods used for this analysis. 
Table 1. Information for USGS sites in the Lake Tahoe Basin, California and Nevada

[Abbreviations: $\mathrm{mi}^{2}$, square miles; $\mathrm{ft}^{3} / \mathrm{s}$, cubic feet per second]

\begin{tabular}{|c|c|c|c|c|c|c|c|c|}
\hline \multicolumn{3}{|r|}{ Gaging station } & \multirow{2}{*}{$\begin{array}{l}\text { Period of record, } \\
\text { water years }\end{array}$} & \multirow{2}{*}{$\begin{array}{c}\text { Drainage } \\
\text { area } \\
\left(\mathrm{mi}^{2}\right)\end{array}$} & \multirow{2}{*}{$\begin{array}{c}\text { 50-year peak } \\
\text { discharge }^{\mathrm{a}} \\
\left(\mathrm{ft}^{3} / \mathrm{s}\right)\end{array}$} & \multirow{2}{*}{$\begin{array}{c}\text { 100-year peak } \\
\text { discharge }^{\mathrm{b}} \\
\left(\mathrm{ft}^{\mathrm{b}} / \mathrm{s}\right)\end{array}$} & \multicolumn{2}{|c|}{ Largest recorded flood peak } \\
\hline $\begin{array}{l}\text { Site no. } \\
\text { (fig. 1) }\end{array}$ & Number & Name & & & & & Date & $\underset{\left(\mathrm{ft}^{3} / \mathrm{s}\right)}{\text { Magnitude }}$ \\
\hline 1 & 10336580 & Upper Truckee River at South Upper Truckee Road near Meyers, Calif. & 1991-2000 & 14.2 & $1,280^{\mathrm{c}}$ & $1,530^{\mathrm{c}}$ & January 2, 1997 & 2,010 \\
\hline 2 & 10336600 & Upper Truckee River near Meyers, Calif. & $1961-86$ & 33.2 & $2,530^{\mathrm{d}}$ & $3,010^{\mathrm{d}}$ & February 1, 1963 & 2,550 \\
\hline 3 & 103366092 & Upper Truckee River at U.S. Highway 50 above Meyers, Calif. & 1991-2000 & 39.3 & $2,890^{\mathrm{c}}$ & $3,450^{\mathrm{c}}$ & January 2, 1997 & 5,120 \\
\hline 4 & 10336610 & Upper Truckee River at South Lake Tahoe, Calif. & 1972-74, 1978, 1980-2000 & 54.0 & $3,650^{\mathrm{d}}$ & $4,560^{\mathrm{d}}$ & January 2, 1997 & 5,480 \\
\hline 5 & 10336630 & Eagle Creek near Camp Richardson, Calif. & 1972-73 & 6.59 & $398^{\mathrm{e}}$ & $514^{\mathrm{e}}$ & November 12, 1973 & 343 \\
\hline 6 & 10336635 & Lake Tahoe tributary near Meeks Bay, Calif. & $1963-73$ & .66 & $53^{\mathrm{f}}$ & $70^{\mathrm{f}}$ & January 31,1963 & 43 \\
\hline 7 & 10336640 & Meeks Creek at Meeks Bay, Calif. & $1972-75$ & 8.11 & $530^{\mathrm{e}}$ & $700^{\mathrm{e}}$ & November 12, 1973 & 526 \\
\hline 8 & 10336645 & General Creek near Meeks Bay, Calif. & 1980-2000 & 7.39 & $930^{\mathrm{d}}$ & $1,120^{\mathrm{d}}$ & January 2,1997 & 797 \\
\hline 9 & 10336650 & Quail Lake Creek at Homewood, Calif. & $1972-74$ & 1.48 & $150^{\mathrm{e}}$ & $207^{\mathrm{e}}$ & May 14,1973 & 24 \\
\hline 10 & 10336655 & Madden Creek near Homewood, Calif. & 1972-73 & 1.67 & $146^{\mathrm{e}}$ & $195^{\mathrm{e}}$ & May 17,1973 & 43 \\
\hline 11 & 10336658 & Madden Creek at Homewood, Calif. & $1972-73$ & 2.04 & $178^{\mathrm{e}}$ & $240^{\mathrm{e}}$ & May 17, 1973 & 86 \\
\hline 12 & 10336660 & Blackwood Creek near Tahoe City, Calif. & 1961-2000 & 11.1 & $2,070^{\mathrm{d}}$ & $2,710^{\mathrm{d}}$ & January 1, 1997 & 2,940 \\
\hline 13 & 10336674 & Ward Creek below confluence near Tahoe City, Calif. & 1992-2000 & 4.84 & $1,260^{\mathrm{c}}$ & $1,660^{\mathrm{c}}$ & January 1, 1997 & 1,220 \\
\hline 14 & 10336675 & Ward Creek at Stanford Rock Trail Crossing near Tahoe City, Calif. & 1992-2000 & 9.18 & $2,110^{\mathrm{c}}$ & $2,780^{\mathrm{c}}$ & January 1, 1997 & 2,370 \\
\hline 15 & 10336676 & Ward Creek at Highway 89 near Tahoe Pines, Calif. & 1973-2000 & 9.73 & $2,210^{\mathrm{d}}$ & $2,910^{\mathrm{d}}$ & January 1, 1997 & 2,530 \\
\hline 16 & 10336684 & Dollar Creek near Tahoe City, Calif. & 1973-74 & 1.07 & $133^{\mathrm{e}}$ & $190^{\mathrm{e}}$ & April 27, 1973 & 32 \\
\hline 17 & 10336686 & Carnelian Creek at Carnelian Bay, Calif. & 1999-2000 & 2.93 & $290^{\mathrm{e}}$ & $390^{\mathrm{e}}$ & May 22, 1999 & 32 \\
\hline 18 & 10336688 & First Creek near Crystal Bay, Nev. & 1970-74, 1991-2000 & 1.07 & $45^{\mathrm{f}}$ & $57^{\mathrm{f}}$ & September 26, 1972 & 22 \\
\hline 19 & 10336690 & Second Creek near Crystal Bay, Nev. & 1970-74 & 1.33 & $122^{\mathrm{e}}$ & $162^{\mathrm{e}}$ & May 18,1970 & 16 \\
\hline 20 & 10336691 & Second Creek at Lakeshore Drive near Crystal Bay, Nev. & 1991-2000 & 1.35 & $125^{\mathrm{e}}$ & $165^{\mathrm{e}}$ & May 31,1995 & 7 \\
\hline 21 & 10336692 & Wood Creek above Jennifer Street near Incline Village, Nev. & 1991-2000 & 1.30 & $59^{\mathrm{f}}$ & $72^{\mathrm{f}}$ & May 1997 & 29 \\
\hline 22 & 10336693 & Wood Creek near Crystal Bay, Nev. & $1967-78$ & 1.69 & $66^{\mathrm{f}}$ & $78^{\mathrm{f}}$ & May 1969 & 40 \\
\hline 23 & 10336694 & Wood Creek at mouth near Crystal Bay, Nev. & 1970-74, 1991-2000 & 1.97 & $75^{\mathrm{c}}$ & $88^{\mathrm{c}}$ & June 26, 1995 & 43 \\
\hline 24 & 103366958 & Third Creek below unnamed tributary near Incline Village, Nev. & 1991-2000 & 4.32 & $146^{\mathrm{C}}$ & $168^{\mathrm{c}}$ & June 4, 1996 & 59 \\
\hline 25 & 10336696 & Third Creek at Incline Village, Nev. & 1970-74 & 4.41 & $149^{\mathrm{c}}$ & $171^{\mathrm{c}}$ & June 19,1971 & 120 \\
\hline 26 & 103366965 & Third Creek at Village Boulevard at Incline Village, Nev. & 1991-2000 & 4.48 & $151^{\mathrm{c}}$ & $173^{\mathrm{c}}$ & June 26, 1995 & 60 \\
\hline 27 & 10336698 & Third Creek near Crystal Bay, Nev. & 1970-73, 1975, 1978-2000 & 6.02 & $191^{\mathrm{f}}$ & $219^{f}$ & June 18,1982 & 150 \\
\hline 28 & 103366993 & Incline Creek above Tyrol Village near Incline Village, Nev. & 1991-2000 & 2.85 & $107^{\mathrm{f}}$ & $130^{\mathrm{f}}$ & $\begin{array}{l}\text { June 26, 1995; } \\
\text { January 2,1997 }\end{array}$ & 52 \\
\hline 29 & 103366995 & Incline Creek at Highway 28 at Incline Village, Nev. & $1990-2000$ & 4.54 & $144^{\mathrm{c}}$ & $177^{\mathrm{c}}$ & January 2, 1997 & 143 \\
\hline 30 & 103366997 & Incline Creek tributary at Country Club Drive at Incline Village, Nev. & 1991-2000 & 1.01 & $43^{\mathrm{c}}$ & $53^{\mathrm{c}}$ & June 26, 1995 & 11 \\
\hline 31 & 10336700 & Incline Creek near Crystal Bay, Nev. & $1970-75,1988-2000$ & 6.69 & $197^{\mathrm{f}}$ & $242^{\mathrm{f}}$ & January 2, 1997 & 179 \\
\hline 32 & 10336725 & Glenbrook Creek at old Highway 50 at Glenbrook, Nev. & 1991-2000 & 3.75 & $139^{\mathrm{c}}$ & $204^{\mathrm{c}}$ & January 2, 1997 & 70 \\
\hline 33 & 10336730 & Glenbrook Creek at Glenbrook, Nev. & $1972-75,1988-2000$ & 4.11 & $150^{\mathrm{f}}$ & $221^{\mathrm{f}}$ & January 2, 1997 & 144 \\
\hline 34 & 10336735 & North Logan House Creek near Glenbrook, Nev. & $1991-2000$ & 1.08 & $32^{\mathrm{f}}$ & $41^{\mathrm{f}}$ & January 2, 1997 & 16 \\
\hline 35 & 10336740 & Logan House Creek near Glenbrook, Nev. & 1984-2000 & 2.09 & $20^{\mathrm{f}}$ & $23^{\mathrm{f}}$ & $\begin{array}{l}\text { January 2, 1997; } \\
\text { June 12, } 1998\end{array}$ & 12 \\
\hline 36 & 10336750 & Edgewood Creek below South Benjamin Drive near Daggett Pass, Nev. & $1991-2000$ & .73 & $46^{\mathrm{f}}$ & $66^{\mathrm{f}}$ & January 1997 & 28 \\
\hline 37 & 10336756 & Edgewood Creek tributary near Daggett Pass, Nev. & $1991-2000$ & .80 & $33^{\mathrm{f}}$ & $47^{\mathrm{f}}$ & January 2, 1997 & 6 \\
\hline 38 & 103367585 & Edgewood Creek at Palisade Drive near Kingsbury, Nev. & 1991-2000 & 3.13 & $114^{\mathrm{f}}$ & $153^{\mathrm{f}}$ & August 14, 1991 & 57 \\
\hline 39 & 10336759 & Edgewood Creek near Stateline, Nev. & $1983-87$ & 3.20 & $116^{\mathrm{c}}$ & $156^{\mathrm{c}}$ & May 27, 1983 & 24 \\
\hline 40 & 103367592 & Eagle Rock Creek near Stateline, Nev. & 1990-2000 & .63 & $57^{\mathrm{e}}$ & $76^{\mathrm{e}}$ & January 2, 1997 & 4 \\
\hline 41 & 10336760 & Edgewood Creek at Stateline, Nev. & 1993-2000 & 5.61 & $-^{\mathrm{g}}$ & $-^{\mathrm{g}}$ & January 2, 1997 & 136 \\
\hline 42 & 10336765 & Edgewood Creek at Lake Tahoe near Stateline, Nev. & 1989-92 & 6.57 & -g & $-\mathrm{g}$ & $\begin{array}{l}\text { March 4, 1991; } \\
\text { October 26, } 1991\end{array}$ & 27 \\
\hline 43 & 10336770 & Trout Creek at U.S. Forest Service Road 12N01 near Meyers, Calif. & $1991-2000$ & 7.41 & $216^{\mathrm{h}}$ & $249^{\mathrm{h}}$ & June 27,1995 & 166 \\
\hline 44 & 10336775 & Trout Creek at Pioneer Trail near South Lake Tahoe, Calif. & $1989,1991-2000$ & 23.1 & $442^{c}$ & $542^{\mathrm{c}}$ & January 2, 1997 & 525 \\
\hline 45 & 10336780 & Trout Creek near Tahoe Valley, Calif. & $1961-2000$ & 36.7 & $638^{\mathrm{d}}$ & $783^{\mathrm{d}}$ & $\begin{array}{l}\text { February 1, 1963; } \\
\text { January 2, } 1997\end{array}$ & 535 \\
\hline 46 & 10336790 & Trout Creek at South Lake Tahoe, Calif. & $1972-74$ & 40.4 & $691^{\mathrm{c}}$ & $848^{\mathrm{c}}$ & May 31,1973 & 190 \\
\hline
\end{tabular}

a. The 50-year peak discharge is theoretical and statistically has a 2-percent chance of occurring in any given year.

b. The 100-year peak discharge is theoretical and statistically has a 1-percent chance of occurring in any given year.

c. Method 5; estimated by methods of Thomas and others (1997, p. 45) using nearby gaging station on the same stream.

d. Method 2; determined from U.S. Interagency Advisory Committee on Water Data (1982) guidelines, considering historic peaks.

e. Method 4; estimated by methods of Thomas and others (1997, p. 45) using the regression equation for the Eastern Sierra.

f. Method 1; determined from U.S. Interagency Advisory Committee on Water Data (1982) guidelines.

g. Affected by regulation; value not determined.

h. Method 3; determined from U.S. Interagency Advisory Committee on Water Data (1982) guidelines for two-station comparison.

\section{Observed Floods}

Table 1 also provides the largest recorded flood peak at each site. During 1997, 31 stream-monitoring sites were in operation, 21 of these sites recorded their largest flood peak as a result of the widespread flooding that occurred on January 1 and 2, 1997, the result of a rain-on-snow event (Hunrichs and others, 1998; and Rowe and others, 1998). For many of the other sites, the largest recorded flood was the result of either convective or frontal rainstorms that occurred during the spring or summer months.

\section{- E. James Crompton, Glen W. Hess, and Rhea P. Williams}

\section{References Cited}

Crippen, J.R., and Pavelka, B.R., 1970, The Lake Tahoe Basin, California-Nevada: U.S. Geological Survey Water-Supply Paper 1972, 56 p.

Hunrichs, R.A., Pratt, D.A., and Meyer, R.W., 1998, Magnitude and frequency of the floods of January 1997 in Northern and Central California-Preliminary determinations: U.S. Geological Survey Open-File Report 98-626, 120 p.

Rowe, T.G., Rockwell, G.L., and Hess, G.W., 1998, Flood of January 1997 in the Lake Tahoe Basin, California and Nevada: U.S. Geological Survey Fact Sheet FS-005-98, 2 p. Thomas, B.E., Hjalmarson, H.W., and Waltemeyer, S.D., 1997, Methods for estimating magnitude and frequency of floods in the southwestern United States: U.S. Geological Survey Water-Supply Paper 2433, 195 p.

U.S. Interagency Advisory Committee on Water Data, 1982, Guidelines for determining flood flow frequency, Bulletin 17-B of the Hydrology Subcommittee: Reston, Va., U.S. Geological Survey, Office of Water Data Coordination, $183 \mathrm{p}$.

For more information on the U.S. Geological Survey studies described in this Fact Sheet, or on other aspects of water resources in Nevada:

Public Information Assistant

U.S. Geological Survey

333 West Nye Lane, Room 203

Carson City, NV 89706-0866
Telephone: (775) 887-7649

Fax: (775) 887-7629

Email: GS-W-NVpublic-info@usgs.gov URL: http://nevada.usgs.gov 